\title{
Activation of STAT3 is involved in malignancy mediated by CXCL12-CXCR4 signaling in human breast cancer
}

\author{
XIAOJIAN LIU ${ }^{1}$, QINGHUAN XIAO $^{1}$, XUEFENG BAI $^{1}$, ZHAOJIN YU $^{1}$, MINGLI SUN $^{1}$, HAISHAN ZHAO $^{1}$, \\ XIAOYI MI ${ }^{2}$, ENHUA WANG ${ }^{2}$, WEIFAN YAO ${ }^{1}$, FENG JIN $^{3}$, LIN ZHAO ${ }^{1}$, JIE REN $^{1}$ and MINJIE WEI ${ }^{1}$ \\ ${ }^{1}$ Department of Pharmacology, China Medical University; ${ }^{2}$ Department of Pathology, College of Basic Medical Sciences, \\ China Medical University; ${ }^{3}$ Department of Surgical Oncology, The First Affiliated Hospital, \\ China Medical University, Shenyang, Liaoning 110001, P.R. China
}

Received July 14, 2014; Accepted September 22, 2014

DOI: $10.3892 /$ or.2014.3536

\begin{abstract}
The chemokine receptor CXCR4 and signal transducer and activator of transcription 3 (STAT3) play an important role in breast cancer malignancy and metastasis. However, it remains unknown whether STAT3 can be activated by CXCR4 in human breast cancer. The expression levels of CXCR4, STAT3 and p-STAT3 in 208 breast cancer tissues and 26 tumor-adjacent tissues were examined by immunohistochemistry. Flow cytometry, western blot analysis and immunoprecipitation were used to study activation of STAT3 by CXCL12-CXCR4 signaling in human breast cancer cell lines. The expression levels of CXCR4, STAT3 and p-STAT3 were higher in the breast cancer samples than these levels in the tumor-adjacent samples. The combined expression of CXCR4 and p-STAT3 was correlated with TNM stage, tumor size, lymph node metastasis and histological grade of breast cancer. In the breast cancer cells, CXCL12 treatment increased the expression of p-STAT3. The CXCR4 antagonist AMD3100 and the Janus kinase 2 (JAK2) antagonist AG490 inhibited the CXCL12-induced increase in the phosphorylation of STAT3. Furthermore, CXCL12 promoted direct binding of JAK2 to CXCR4. Our findings suggest that activation of the JAK2/ STAT3 pathway via CXCL12-CXCR4 signaling plays an important role in breast cancer malignancy and metastasis. Targeting the CXCL12-CXCR4/JAK2/STAT3 signaling pathway may be a potential therapeutic strategy for the treatment of breast cancer.
\end{abstract}

\section{Introduction}

Breast cancer is one of the most common malignant cancers in women, and is one of the leading causes of cancer-related deaths worldwide. Distant metastasis is the main cause of

Correspondence to: Professor Minjie Wei, Department of Pharmacology, China Medical University, Shenyang, Liaoning 110001, P.R. China

E-mail: weiminjiecmu@163.com

Key words: chemokine receptor, CXCR4, STAT3, CXCL12, breast cancer breast cancer-related mortality (1). However, the mechanisms leading to breast cancer metastasis remain poorly understood. Breast cancer commonly metastasizes to certain organs such as the lymph node, bone marrow, and lung, which exhibit enhanced secretion of cytokines, but rarely to other organs with low expression of cytokines, such as the kidney and skin. This characteristic feature highlights the important role played by chemokines and their receptors in breast cancer metastasis $(2,3)$.

CXCR4 and its ligand CXCL12 (also called SDF-1 $\alpha$ ) play a critical role in breast cancer carcinogenesis and metastasis, and targeting the CXCL12-CXCR4 signaling pathway is a potential therapeutic strategy for the treatment of breast cancer $(3,4)$. Activation of CXCL12-CXCR4 signaling has been reported to promote survival, proliferation, adhesion, chemotaxis and migration of breast cancer cells (5). The binding of CXCL12 to CXCR4 results in activation of several signaling pathways in breast cancer including the MAPK/ ERK1/2 $(6,7)$ and PI3K/AKT $(8,9)$ pathways. It has been reported that CXCL12-CXCR4 signaling activates the Janus kinase 2 (JAK2) signal transducer and activator of transcription 3 (STAT3) pathway $(10,11)$, and STAT3 inactivation inhibits murine breast cancer metastasis (12). However, it remains unclear whether activation of the JAK2/STAT3 pathway is involved in the CXCL12-CXCR4 signaling axis in human breast cancer.

STAT3, a transcription factor that belongs to the STAT family, has been found to be overexpressed and constitutively activated in many malignant tumors (13-15). STAT3 has been regarded as an oncogene, and constitutive activation of STAT3 can lead to abnormal cell proliferation and malignant transformation $(16,17)$. JAK2 can recruit and activate STAT3, which translocates to the nucleus and regulates transcription of a variety of genes that are associated with proliferation, differentiation, apoptosis and metastasis of many types of cancer cells $(18,19)$. However, it remains unclear whether the JAK2/STAT3 pathway is coupled to CXCL12-CXCR4 signaling. It has been reported that in response to CXCL12, CXCR4 activates the JAK2/STAT3 pathway $(10,11,20)$. However, Moriguchi et al reported that CXCL12-CXCR4 signaling is independent of the JAK2/STAT3 pathway in primary lymphocytes (21). Furthermore, STAT3 activation 
is associated with the CXCR4 signaling pathway in many types of cancers such as cervical carcinoma (22), small cell lung cancer (23) and bladder cancer (24). However, Lee et al reported that CXCL12 failed to activate STAT3 in gastric cancer cells (25). Constitutively activated STAT3 was found in primary tumors from high risk breast cancer patients (26), and is associated with breast cancer growth and metastasis in xenograft models $(27,28)$. However, it remains unclear whether CXCL12-CXCR4 signaling is coupled to the JAK2/STAT3 pathway in human breast cancer.

In the present study, we aimed to investigate the association between CXCR4 and STAT3, particularly p-STAT3, in breast cancer tissues from Chinese patients, and to identify the JAK2/STAT3 pathway in CXCL12-CXCR4 signaling in human breast cell lines. We performed immunohistochemistry to examine the expression of CXCR4, STAT3 and p-STAT3 in 208 patients with breast cancer, and analyzed their correlation with clinicopathological characteristics of these patients. We found that the expression levels of CXCR4 and STAT3, particularly p-STAT3, were increased with increased TNM stage and lymph node metastasis. The combined expression of both CXCR4 and p-STAT3 was correlated with breast cancer malignancy. Furthermore, we found that inhibition of CXCR4 or JAK2 prevented CXCL12-induced phosphorylation of STAT3 in breast cancer cell lines. Our results suggest that activation of the JAK2/STAT3 pathway by CXCL12-CXCR4 signaling may play an important role in breast cancer malignancy, and targeting the CXCL12-CXCR4/JAK2/STAT3 signaling pathway may be a potential therapeutic strategy for the treatment of breast cancer.

\section{Materials and methods}

Patients and tissue samples. The Ethics Committee of China Medical University approved this study. Human breast cancer samples of 208 female patients with primary breast cancer were collected at the Department of Surgical Oncology and The Department of General Surgery of the First Affiliated Hospital of China Medical University between 2003 and 2010. Some of the samples were used in our previous studies $(29,30)$. The median age of the breast cancer patients was 51 years (range, 29 to 85 years). Of the 208 patients, the stage and the histological grading of the cancer were evaluated according to the TNM staging system and the Elston-Ellis modification of Scarff-Bloom-Richardson grading system, respectively. Clinicopathological data including patient age, menopausal status, tumor size, tumor type, lymph node metastasis, and the status of the estrogen receptor (ER), progesterone receptor (PR) and human epidermal growth factor receptor (HER2) were retrospectively retrieved from the medical records. Of the 208 patients, 182 patients had invasive ductal carcinoma, and 10 had invasive lobular carcinoma. Other patients presenting with tumors of less incidence were categorized into one group, including 3 patients with cribriform carcinoma, 3 patients with micropapillary carcinoma, 2 patients with mucinous carcinoma, 2 patients with medullary carcinoma, 2 patients with papillary carcinoma, 1 patient with tubular carcinoma and 3 patients with ductal carcinoma in situ. This study included 26 tumor-adjacent samples as controls. The breast tissues outside the cancer loci were selected as tumor-adjacent samples. The diagnosis of breast cancer was confirmed by pathological staining. The tumor-adjacent samples exhibited no tumor texture histologically. All patients did not undergo radiation therapy, chemotherapy and hormonal therapy.

Cell culture. Human breast cancer cell lines (MDA-MB231, BT-549, and MCF-7) were obtained from the American Type Culture Collection (ATCC), and maintained according to ATCC's recommendation. To investigate the effect of CXCR4 on the phosphorylation of STAT3, human breast cancer cells were treated with $100 \mathrm{ng} / \mathrm{ml}$ CXCL12 (350-NF/ CF; R\&D Systems Inc.) for 0, 2, 5, 15 and 30 min after serum-starvation for $4 \mathrm{~h}$. To investigate whether CXCR4 or JAK2 mediates activation of STAT3 by CXCL12, cells were pretreated with the CXCR4 antagonist AMD3100 $(10 \mu \mathrm{M}$, A5602; Sigma-Aldrich St. Louis, MO, USA) or the JAK2 inhibitor AG490 (25 $\mu \mathrm{M}, 658401$; Calbiochem) for $2 \mathrm{~h}$ before treatment with CXCL12.

Immunohistochemistry. Tissue sections (4- $\mu$ m thick) were obtained from formalin-fixed and paraffin-embedded tissue blocks from the control and breast cancer samples. Sections were washed in xylene to remove the paraffin, and rehydrated with serial dilutions of alcohol, followed by a wash in PBS solution. Endogenous peroxidase activity was blocked by $3 \%$ hydrogen peroxide in methanol at $37^{\circ} \mathrm{C}$ for $20 \mathrm{~min}$. Sections were then incubated with primary antibodies against CXCR4 (1:100 dilution, ab2074; Abcam), STAT3 (1:50 dilution, \#4904; Cell Signaling Technology) and p-STAT3 (Tyr705) (1:200 dilution, \#9145; Cell Signaling Technology) overnight at $4^{\circ} \mathrm{C}$. After the primary antibody was washed off, sections were incubated with goat anti-rabbit biotin-conjugated secondary antibodies (1:1000 dilution; Dako, Glostrup, Denmark) for $30 \mathrm{~min}$ at $37^{\circ} \mathrm{C}$. The tissue sections were then incubated with streptavidin horseradish peroxidase for $30 \mathrm{~min}$ at $37^{\circ} \mathrm{C}$. DAB (3,3-diaminobenzidine) substrate was applied to the sections, and then sections were counterstained with hematoxylin. Sections in which primary antibodies were omitted were used as negative control.

Immunostaining was examined under a light microscope by two pathologists blinded to the experimental conditions. Agreement on the scores between the two pathologists was nearly $100 \%$. In cases in which the pathologists disagreed to the score, the immunohistochemical scoring was repeated by both pathologists until the same score was achieved. The immunoreactivity was evaluated using a scoring system according to the percentage of stained cells and the intensity of the immunoreactivity. The intensity of immunoreactivity was scored as follows: 0 for no staining, 1 for weak staining, 2 for moderate staining, and 3 for strong staining. The percentage of stained cells was scored as follows: 0 for $<10 \%, 1$ for $10-29 \%$, 2 for $30-49 \%, 3$ for $50-74 \%$, and 4 for $\geq 75 \%$. The final immunoreactive score was determined by multiplying the intensity score with the score for the percentage of positively stained cells. The minimum score was 0 and the maximum score was 12. Low and high expression of CXCR4, STAT3 and p-STAT3 was defined by a final score of $<6$ and $\geq 6$, respectively.

Flow cytometry. Flow cytometric analysis was performed on a FACSCalibur (Becton-Dickinson, Franklin Lakes, NJ, USA). 


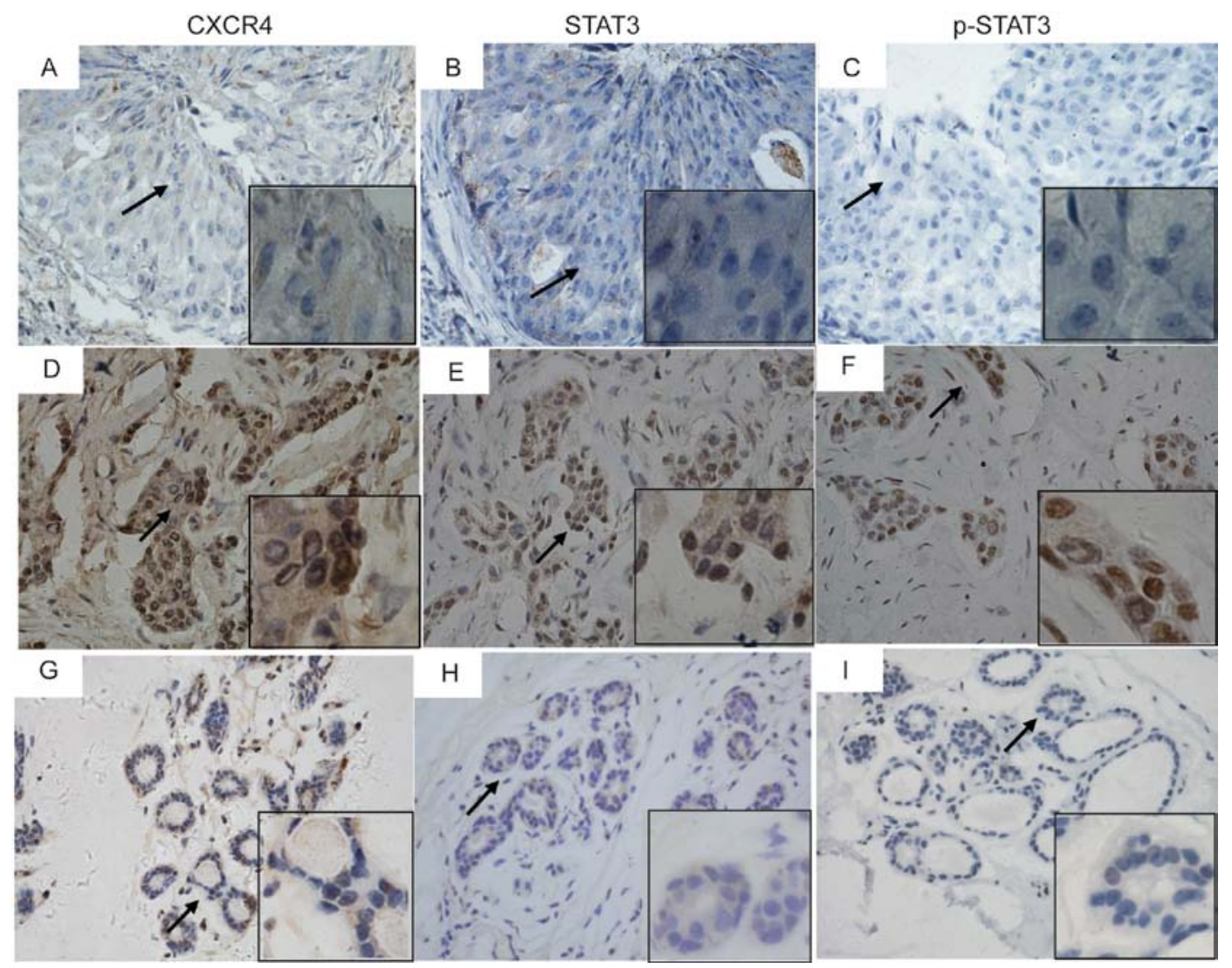

Figure 1. Immunohistochemical staining of CXCR4 (A, D and G), STAT3 (B, E and H), and phospho-STAT3 (Tyr705) (C, F and I) in breast cancer tissues (A-F) and tumor-adjacent tissues (G-I). Representative micrographs of low expression (A-C), and high expression (D-F) of CXCR4, STAT3 and p-STAT3 in consecutive sections of breast cancer tissues, and the expression of CXCR4, STAT3, and p-STAT3 in tumor-adjacent tissues (G-I). Magnification; x400. Areas indicated by an arrow are magnified in inserts $(\mathrm{x} 1,000)$.

Cells $\left(1 \times 10^{6}\right.$ cells $\left./ \mathrm{ml}\right)$ were fixed with $4 \%$ paraformaldehyde, and then washed and resuspended in PBS containing $0.5 \%$ bovine serum albumin. Cells were permeabilized with $0.1 \%$ Triton-X 100, and incubated with the primary antibodies against CXCR4 (1:100 dilution) for $30 \mathrm{~min}$ at room temperature. Isotype rabbit IgG was used as a control. After washing, cells were labeled with Alexa Fluor 488-conjugated secondary antibodies (Molecular Probes, A21206; Invitrogen) for $1 \mathrm{~h}$ at $4^{\circ} \mathrm{C}$. Cells were subsequently analyzed by flow cytometry.

Western blot analysis. Human breast cancer cells were homogenized on ice in RIPA lysis buffer (Beyotime, Nantong, China) containing a cocktail of protease and phosphatase inhibitors (Sigma-Aldrich). Proteins were resolved by SDS-PAGE, and transferred onto polyvinylidene fluoride membranes by electroblotting. The membranes were incubated with primary antibodies against CXCR4 (1:2,000 dilution), STAT3 (1:4,000 dilution) and p-STAT3 (1:2,000 dilution) at $4^{\circ} \mathrm{C}$ with gentle shaking overnight. GAPDH was used as a loading control. The membranes were then incubated with horseradish peroxidaselinked goat anti-rabbit secondary antibodies (dilution 1:5,000) at room temperature for $2 \mathrm{~h}$. Bands were visualized using a chemiluminescence detection system.

Immunoprecipitation. MCF-7 cells were solubilized on ice with lysis buffer $(150 \mathrm{mM} \mathrm{NaCl}, 10 \mathrm{mM}$ Tris-HCl, $\mathrm{pH} 7.5$, $1 \%$ Triton X-100) supplemented with a cocktail of phosphatase and proteinase inhibitors (Sigma-Aldrich). Lysates were centrifuged at $10,000 \mathrm{x}$ g for $15 \mathrm{~min}$ at $4^{\circ} \mathrm{C}$. The supernatants were incubated with antibodies against CXCR4 (1:100 dilution) or non-specific rabbit IgG (control), and protein A-agarose at $4^{\circ} \mathrm{C}$ overnight. The immunoprecipitates were collected by centrifugation, and the agarose pellet was suspended in $2 \mathrm{X}$ sodium dodecyl sulphate-polyacrylamide gel electrophoresis (SDS-PAGE) buffer. The expression of JAK2 was determined by western blotting, using antibodies against JAK2 (1:1,000 dilution, \#3230; Cell Signaling Technology).

Statistical analysis. Statistical analyses were performed using SPSS 11.5. The numerical data are presented as mean and standard deviation. Student t-test or one-way analysis of variance (ANOVA) was used to compare the difference in the means among two or more groups, respectively. Categorical data were compared with Pearson Chi squared tests. The Spearman's correlation analysis was applied to assess the association of the expression of CXCR4 with the expression of STAT3 and p-STAT3. Probability (P)-values $<0.05$ were considered to indicate statistically significant results.

\section{Results}

Expression of CXCR4, STAT3 and p-STAT3 in the breast cancer tissues. The clinicopathological characteristics of 208 patients with breast cancer are shown in Table I. Of the 208 breast cancer patients, the TNM stage, tumor size, lymph node metastasis, and histological grading were recorded in 194, 193, 
Table I. Correlation of the expression of CXCR4, STAT3 and p-STAT3 with the clinicopathological parameters of the breast cancer cases.

\begin{tabular}{|c|c|c|c|c|c|c|c|c|c|c|}
\hline \multirow[b]{2}{*}{ Parameters } & \multirow[b]{2}{*}{$\mathrm{N}^{\mathrm{a}} / 208$} & \multicolumn{2}{|c|}{$\begin{array}{c}\text { CXCR4 expression } \\
\mathrm{n}(\%)\end{array}$} & \multirow[b]{2}{*}{ P-value ${ }^{b}$} & \multicolumn{2}{|c|}{$\begin{array}{c}\text { STAT3 expression } \\
\mathrm{n}(\%)\end{array}$} & \multirow[b]{2}{*}{ P-value ${ }^{b}$} & \multicolumn{2}{|c|}{$\begin{array}{c}\text { p-STAT3 expression } \\
\mathrm{n}(\%)\end{array}$} & \multirow[b]{2}{*}{$\mathrm{P}$-value } \\
\hline & & Low & High & & Low & High & & Low & High & \\
\hline Age (years) & $208 / 208$ & & & 0.563 & & & 0.749 & & & 0.189 \\
\hline$<50$ & 86 & $27(31.4)$ & $59(68.6)$ & & $25(29.1)$ & $61(70.9)$ & & $53(61.6)$ & $33(38.4)$ & \\
\hline$\geq 50$ & 122 & $43(35.2)$ & $79(64.8)$ & & $33(27.0)$ & $89(73.0)$ & & $64(52.5)$ & $58(47.5)$ & \\
\hline Menopause status & $196 / 208$ & & & 0.590 & & & 0.160 & & & 0.080 \\
\hline Pre-menopausal & 83 & $31(37.3)$ & $52(62.7)$ & & $29(34.9)$ & $54(65.1)$ & & $53(63.9)$ & $30(36.1)$ & \\
\hline Post-menopausal & 113 & $38(33.6)$ & $75(66.4)$ & & $29(25.7)$ & $84(74.3)$ & & $58(51.3)$ & $55(48.7)$ & \\
\hline Tumor type & $208 / 208$ & & & 0.850 & & & 0.080 & & & 0.970 \\
\hline Ductal & 182 & $60(33.0)$ & $122(67.0)$ & & $46(25.3)$ & $136(74.7)$ & & $102(56.0)$ & $80(44.0)$ & \\
\hline Lobular & 10 & $4(40.0)$ & $6(60.0)$ & & $5(50.0)$ & $5(50.0)$ & & $6(60.0)$ & $4(40.0)$ & \\
\hline Others & 16 & $6(37.5)$ & $10(62.5)$ & & $7(43.8)$ & $9(56.2)$ & & $9(56.2)$ & $7(43.8)$ & \\
\hline TNM stage & $194 / 208$ & & & 0.002 & & & 0.045 & & & 0.000 \\
\hline $\mathrm{I}$ & 29 & $16(64.7)$ & $13(35.3)$ & & $11(37.9)$ & $18(62.1)$ & & $21(72.4)$ & $8(27.6)$ & \\
\hline II & 100 & $38(38.0)$ & $62(62.0)$ & & $32(32.0)$ & $68(68.0)$ & & $65(65.0)$ & $35(35.0)$ & \\
\hline III-IV & 65 & $13(20.0)$ & $52(80.0)$ & & $11(16.9)$ & $54(83.1)$ & & $22(33.8)$ & $43(66.2)$ & \\
\hline Tumor size $(\mathrm{cm})$ & $193 / 208$ & & & 0.193 & & & 0.999 & & & 0.080 \\
\hline$\leq 2.0$ & 53 & $23(43.4)$ & $30(56.6)$ & & $15(28.3)$ & $38(71.7)$ & & $31(58.5)$ & $22(41.5)$ & \\
\hline$>2.0$ to $\leq 5.0$ & 98 & $32(33.5)$ & $66(64.5)$ & & $28(28.6)$ & $70(71.4)$ & & $62(63.3)$ & $36(36.7)$ & \\
\hline$>5.0$ & 42 & $11(26.2)$ & $31(73.8)$ & & $12(28.6)$ & $30(71.4)$ & & $18(42.9)$ & $24(57.1)$ & \\
\hline LNM & $186 / 208$ & & & 0.025 & & & 0.040 & & & 0.023 \\
\hline No & 78 & $34(43.6)$ & $44(56.4)$ & & $28(35.9)$ & $50(64.1)$ & & $50(64.1)$ & $28(35.9)$ & \\
\hline Yes & 108 & $30(27.8)$ & $78(72.2)$ & & $24(22.2)$ & $84(77.8)$ & & $51(51.0)$ & $57(57.0)$ & \\
\hline Histological grade & $177 / 208$ & & & 0.277 & & & 0.072 & & & 0.000 \\
\hline I & 25 & $10(40.0)$ & $15(60.0)$ & & $11(44.0)$ & $14(56.0)$ & & $22(88.0)$ & $3(12.0)$ & \\
\hline II & 131 & $46(35.1)$ & 85 (64.9) & & 35 (26.7) & $96(73.3)$ & & $71(54.2)$ & $60(45.8)$ & \\
\hline III & 21 & $4(19.0)$ & $17(81.0)$ & & $3(14.3)$ & $18(85.7)$ & & $6(28.6)$ & $15(71.4)$ & \\
\hline ER & $179 / 208$ & & & 0.640 & & & 0.964 & & & 0.312 \\
\hline Negative & 68 & $25(36.8)$ & $43(63.2)$ & & $20(29.4)$ & $48(70.6)$ & & $42(61.8)$ & $26(38.2)$ & \\
\hline Positive & 111 & $37(33.3)$ & 74 (66.7) & & 33 (29.7) & $78(70.3)$ & & $60(54.1)$ & $51(45.9)$ & \\
\hline PR & $179 / 208$ & & & 0.415 & & & 0.978 & & & 0.752 \\
\hline Negative & 72 & $22(30.6)$ & $50(69.4)$ & & $21(29.2)$ & $51(70.8)$ & & $40(55.6)$ & $32(44.4)$ & \\
\hline Positive & 107 & $39(36.4)$ & $68(63.6)$ & & $31(29.0)$ & $76(71.0)$ & & $62(57.9)$ & $45(42.1)$ & \\
\hline HER 2 & $164 / 208$ & & & 0.307 & & & 0.852 & & & 0.584 \\
\hline Negative & 53 & $21(39.6)$ & $32(60.4)$ & & $17(32.1)$ & $36(67.9)$ & & $32(60.4)$ & $21(39.6)$ & \\
\hline Positive & 111 & $35(31.5)$ & $76(68.5)$ & & $34(30.6)$ & 77 (69.4) & & $62(55.9)$ & $49(44.1)$ & \\
\hline
\end{tabular}

${ }^{a}$ Number of cases for which corresponding data are available. ${ }^{b}$ P-value obtained from Pearson Chi-square test. LNM, lymph node metastasis; ER, estrogen receptor; PR, progesterone receptor; HER2, human epidermal growth factor receptor 2; STAT3, signal transducer and activator of transcription 3.

186 and 177 patients, respectively. ER, PR, and HER2 were examined in 179, 179 and 164 patients, respectively.

We studied the expression of CXCR4, STAT3, and p-STAT3 in 208 tumor samples and in 26 tumor-adjacent samples from patients with breast cancer, using immunohistochemistry (Fig. 1). The expression levels of CXCR4, STAT3, and p-STAT3 were higher in the breast cancer samples than these levels in the tumor-adjacent samples (Fig. 2). CXCR4 immunoreactivity showed low expression and high expression in $70(33.6 \%)$ and $138(66.4 \%)$ of the 208 breast cancer samples, respectively, and in 19 (70.1\%) and 7 (26.9\%) of the 26 tumor-adjacent samples, respectively (Fig. 2, $\mathrm{P}<0.001$ ). The expression level of CXCR4 increased with increased TNM stage $(\mathrm{P}=0.025)$ and lymph node metastasis $(\mathrm{P}=0.039)$, but not with tumor size $(\mathrm{P}=0.193)$ and histological grade $(\mathrm{P}=0.277)$ (Table I). STAT3 immunoreactivity showed low expression 
Table II. Correlation of the combined expression of CXCR4/STAT3 or CXCR4/pSTAT3 with TNM stage, tumor size, metastasis and histological grade in the breast cancer cases.

\begin{tabular}{|c|c|c|c|c|c|c|c|c|}
\hline \multirow[b]{2}{*}{ Parameters } & \multicolumn{3}{|c|}{ CXCR4/STAT3 expression, $\mathrm{n}(\%)$} & \multirow[b]{2}{*}{ P-value ${ }^{b}$} & \multicolumn{3}{|c|}{ CXCR4/p-STAT3 expression, n (\%) } & \multirow[b]{2}{*}{$\mathrm{P}$-value } \\
\hline & Low/Low & $\begin{array}{l}\text { Low/High or } \\
\text { High/Low }\end{array}$ & High/High & & Low/Low & $\begin{array}{l}\text { Low/High or } \\
\text { High/Low }\end{array}$ & High/High & \\
\hline \multicolumn{9}{|l|}{ TNM stage } \\
\hline I & $7(24.1)$ & $14(48.3)$ & $8(27.6)$ & 0.002 & $12(41.4)$ & $13(44.8)$ & $4(13.8)$ & $<0.001$ \\
\hline II & $18(18.0)$ & $34(34.0)$ & $48(48.0)$ & & $29(29.0)$ & $45(45.0)$ & $26(26.0)$ & \\
\hline III-IV & $5(7.7)$ & $14(21.5)$ & $46(70.8)$ & & $10(15.4)$ & $17(26.2)$ & $38(58.5)$ & \\
\hline \multicolumn{9}{|c|}{ Tumor size $(\mathrm{cm})$} \\
\hline$\leq 2.0$ & $10(18.9)$ & $20(37.7)$ & $23(43.4)$ & 0.348 & $16(30.2)$ & $24(45.3)$ & $13(24.5)$ & 0.043 \\
\hline$>2.0$ to $<4.0$ & $13(13.3)$ & $34(34.7)$ & $51(52.0)$ & & $25(25.5)$ & $43(43.9)$ & $30(30.6)$ & \\
\hline$\geq 4.0$ & 7 (16.7) & $9(21.4)$ & $26(61.9)$ & & $10(23.8)$ & $10(23.8)$ & $22(52.4)$ & \\
\hline \multicolumn{9}{|l|}{ LNM } \\
\hline No & $15(19.2)$ & $33(42.3)$ & $30(38.5)$ & 0.002 & $24(30.8)$ & $35(44.9)$ & $19(24.4)$ & 0.013 \\
\hline Yes & 15 (13.9) & $24(22.2)$ & 69 (63.9) & & $25(23.1)$ & $34(31.5)$ & $49(45.4)$ & \\
\hline \multicolumn{9}{|l|}{$\begin{array}{l}\text { Histological } \\
\text { grade }\end{array}$} \\
\hline I & $7(28.0)$ & $8(32.0)$ & $10(40.0)$ & 0.078 & $10(40.0)$ & $12(48.0)$ & $3(12.0)$ & 0.027 \\
\hline II & $19(14.5)$ & $44(33.6)$ & $68(51.9)$ & & $33(25.2)$ & $52(35.7)$ & $46(35.1)$ & \\
\hline III & 1 & $4(19.0)$ & $16(76.2)$ & & $3(14.3)$ & $6(28.6)$ & $12(57.1)$ & \\
\hline
\end{tabular}

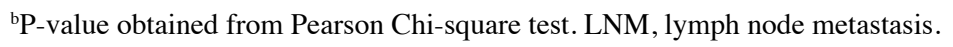

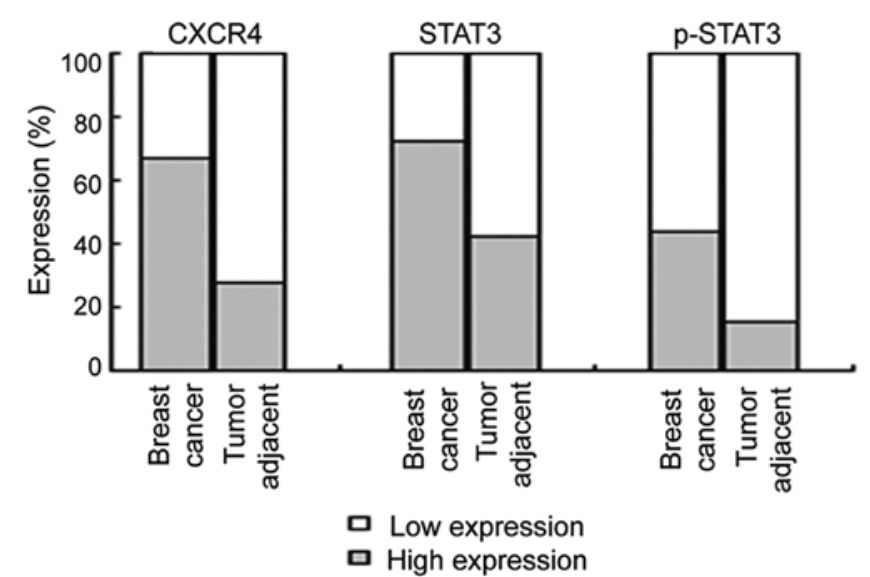

Figure 2. The low and high expression of CXCR4, STAT3 and p-STAT3 in breast cancer samples and tumor-adjacent samples. The expression levels of CXCR4, STAT3 and p-STAT3 in breast cancer samples were significantly higher than levels in the tumor-adjacent samples (Pearson Chi-square; $\mathrm{P}<0.001$ for CXCR4, $\mathrm{P}=0.002$ for $\mathrm{STAT} 3, \mathrm{P}=0.003$ for $\mathrm{p}$-STAT3).

and high expression in $58(27.9 \%)$ and $150(72.1 \%)$ of the 208 breast cancer samples, respectively and in $15(57.7 \%)$ and 11 (42.3\%) of 26 tumor-adjacent samples, respectively (Fig. 2, $\mathrm{P}=0.002)$. The expression level of STAT3 increased with increased TNM stage $(\mathrm{P}=0.045)$ and lymph node metastasis $(\mathrm{P}=0.040)$, but not with the tumor size $(\mathrm{P}=0.999)$ and histological grade $(\mathrm{P}=0.072)$ (Table I). $\mathrm{p}$-STAT3 immunoreactivity showed low expression and high expression in 117 (56.2\%) and
$91(43.8 \%)$ of the 208 breast cancer samples, respectively and in $22(84.6 \%)$ and $4(15.4 \%)$ of the 26 tumor-adjacent samples, respectively (Fig. 2, $\mathrm{P}=0.003$ ). The expression of $\mathrm{p}$-STAT3 was increased with increased TNM stage $(\mathrm{P}<0.001)$, lymph node metastasis $(\mathrm{P}=0.023)$ and histological grade $(\mathrm{P}<0.001)$, but not with the tumor size $(\mathrm{P}=0.080)$ (Table $\mathrm{I})$. In addition, the expression levels of CXCR4, STAT3, and p-STAT3 did not significantly differ in regards to patient age, menstruation status, tumor type, ER status, PR status and HER2 status.

Association of the combined expression of CXCR4/STAT3 and CXCR4/p-STAT3 with clinicopathological characteristics of the breast cancer. We further investigated the correlation of the expression of CXCR4 with the expression of STAT3 and p-STAT3. A positive correlation was observed between the expression of CXCR4 and STAT3 $(r=0.260, \mathrm{P}<0.001)$ and between the expression of CXCR4 and p-STAT3 $(r=0.300$, $\mathrm{P}<0.001$ ). These results suggest that the signaling pathway that involves CXCR4 and STAT3 may play a role in breast cancer carcinogenesis.

We then investigated the association of the combined expression of CXCR4 and STAT3 (CXCR4/STAT3) or CXCR4 and p-STAT3 (CXCR4/p-STAT3) with the clinicopathological characteristics of the breast cancer cases (Table II). Compared with the combined low expression of CXCR4 and STAT3 (Low/Low) and the high expression of either CXCR4 or STAT3 (High/Low or Low/High), the combined high expression of CXCR4/STAT3 (High/High) was highly correlated with TNM stage $(\mathrm{P}=0.002)$ and lymph node metastasis $(\mathrm{P}=0.002)$, but not with the tumor size $(\mathrm{P}=0.348)$ and histological grade 

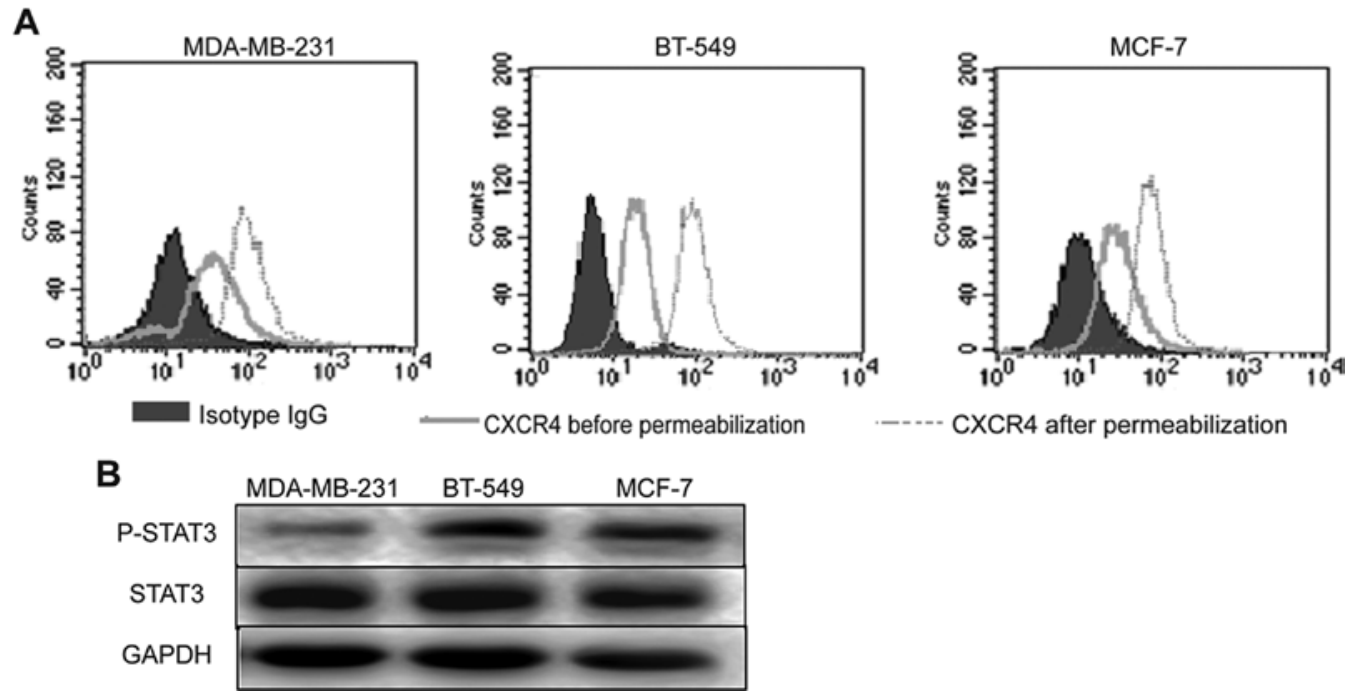

Figure 3. Endogenous expression of CXCR4, STAT3 and p-STAT3 in breast cancer cell lines. (A) Flow cytometric analysis of MDA-MB-231, BT-549 and MCF-7 cells. The cell surface expression of CXCR4 was analyzed using anti-CXCR4 antibodies. The total expression of CXCR4 was detected after cells were permeabilized. (B) Representative western blot analysis showing the expression of STAT3 and p-STAT3 in MDA-MB-231, BT-549 and MCF-7 cells. The experiments were repeated three times.

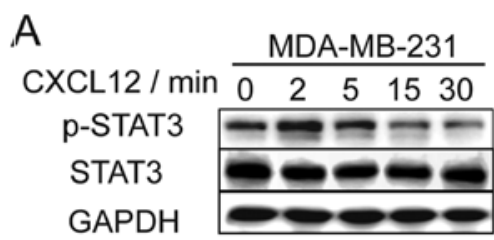

B

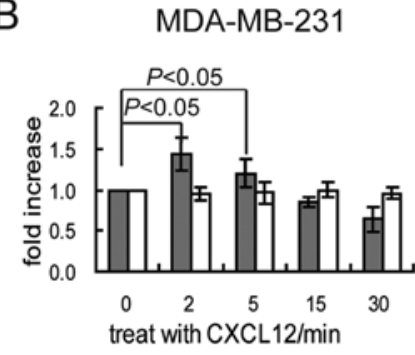

C

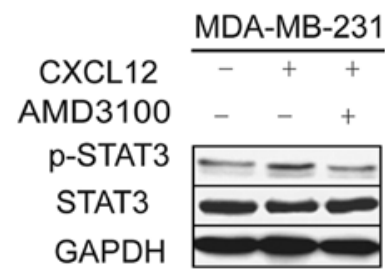

D

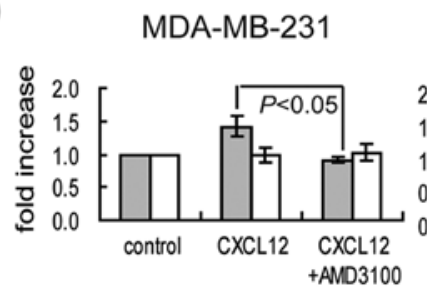

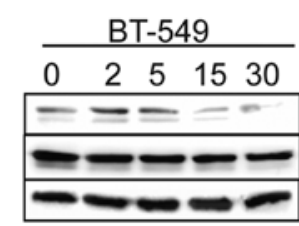

BT-549

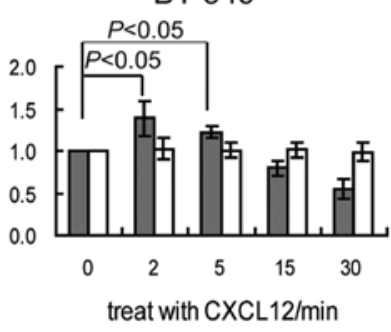

口p-STAT3

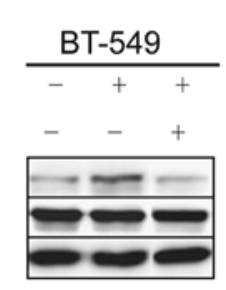

BT-549

口STAT3

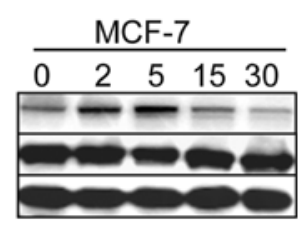

MCF-7
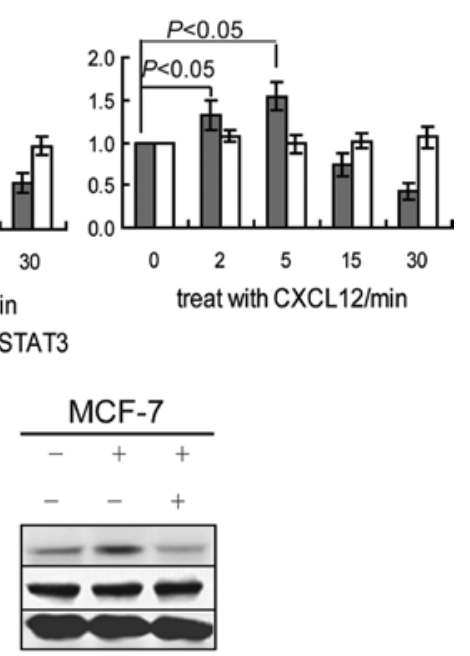

MCF-7

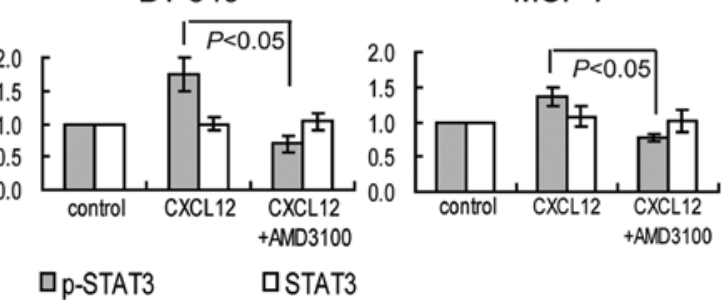

Figure 4. Activation of STAT3 by CXCL12 in breast cancer cells. (A) Representative western blot analysis showing the expression of STAT3 and p-STAT3 in MDA-MB-231, BT-549 and MCF-7 cells treated with CXCL12 (100 ng/ml) for 0, 2, 5, 15 and $30 \mathrm{~min}$. GAPDH was used as a loading control. (B) Quantification of p-STAT3 and STAT3 expression normalized to GAPDH; $n=3$. (C) Representative western blot analysis showing the expression of STAT3 and p-STAT3 in MDA-MB-231, BT-549 and MCF-7 cells treated with CXCL12 (100 ng/ml) in the presence or absence of AMD3100. Cells were treated with AMD3100 $(10 \mu \mathrm{M})$ for $2 \mathrm{~h}$ followed by stimulation with CXCL12 for 2 min (MDA-MB-231 and BT-549) or 5 min (MCF-7). GAPDH was used as a loading control. (D) Quantification of $\mathrm{p}-\mathrm{STAT} 3$ and STAT3 expression normalized to GAPDH; $\mathrm{n}=3$. 

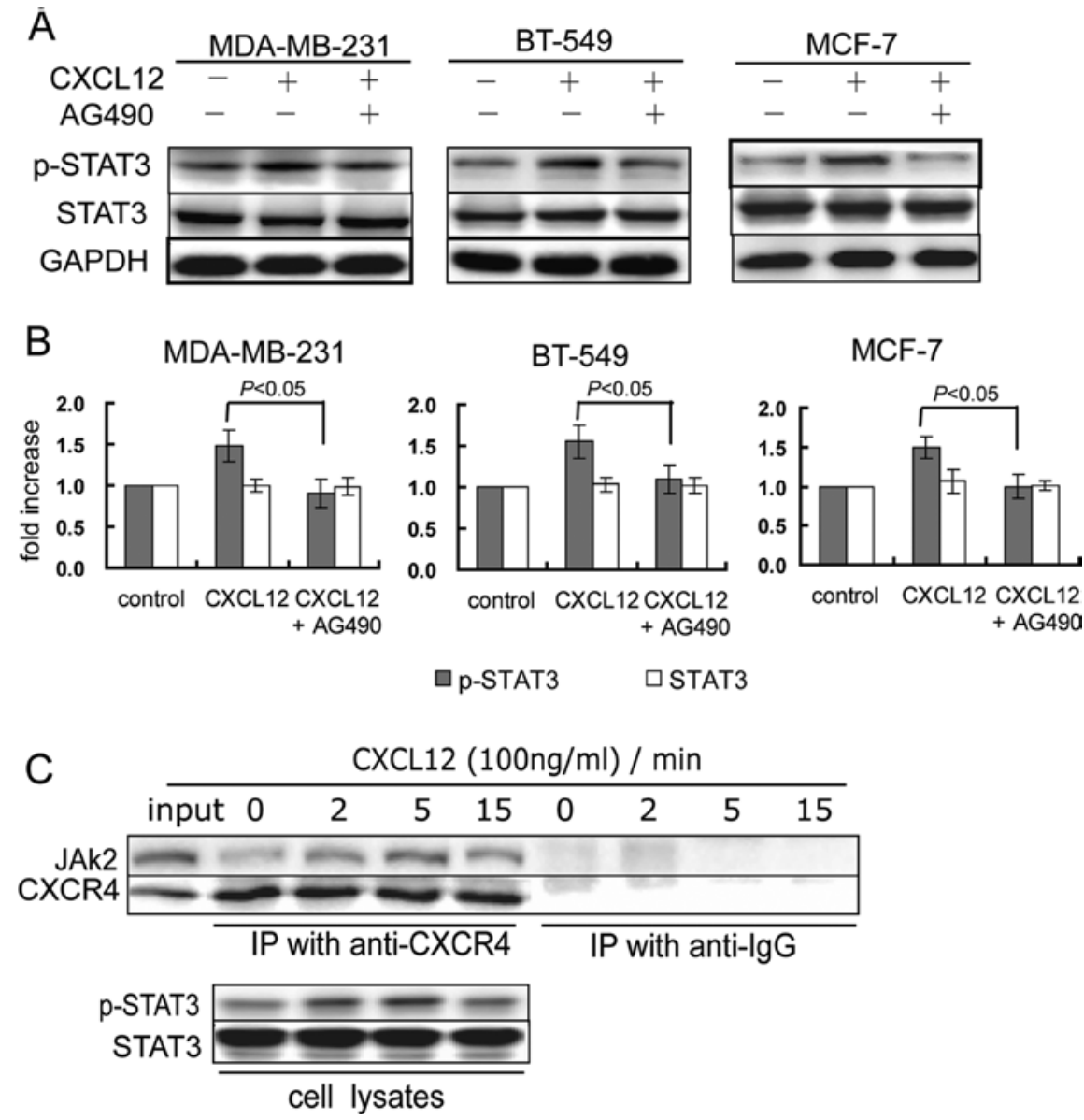

Figure 5. CXCL12-CXCR4 signaling activates the JAK2/STAT3 pathway. (A) Representative western blot analysis showing the expression of STAT3 and p-STAT3 in MDA-MB-231, BT-549 and MCF-7 cells treated with CXCL12 $(100 \mathrm{ng} / \mathrm{ml})$ in the presence or absence of AG490. Cells were treated with AG490 $(25 \mu \mathrm{M})$ for $2 \mathrm{~h}$ followed by stimulation with CXCL12 for $2 \mathrm{~min}$ (MDA-MB-231 and BT-549) or 5 min (MCF-7). GAPDH was used as a loading control. (B) Quantification of p-STAT3 and STAT3 expression normalized to GAPDH; $\mathrm{n}=3$. (C) Immunoprecipitation detecting the direct binding of Janus kinase 2 (JAK2) to CXCR4. MCF-7 cells were treated with CXCL12 $(100 \mathrm{ng} / \mathrm{ml})$ for 0, 2, 5 or $15 \mathrm{~min}$. Cell lysates were immunoprecipitated with CXCR4 antibodies or istotype IgG, and the expression of JAK2 was determined by the western blot analysis. The expression of p-STAT3 and STAT3 in cell lysates was detected by western blot analysis. The experiments were repeated three times.

$(\mathrm{P}=0.078)$. Compared with the combined low expression of CXCR4 and p-STAT3 (Low/Low) and the high expression of either CXCR4 or p-STAT3 (High/Low or Low/High), the combined high expression of CXCR4/p-STAT3 (High/High) was highly correlated with TNM stage $(\mathrm{P}<0.001)$, tumor size $(\mathrm{P}=0.043)$, lymph node metastasis $(\mathrm{P}=0.013)$ and histological grade $(\mathrm{P}=0.027)$.

CXCR4-mediated activation of JAK2/STAT3 in human breast cancer cell lines. The positive correlation of CXCR4 expression with the expression level of STAT3 and p-STAT3 in breast cancer suggests that p-STAT3 may be regulated by CXCR4. To investigate the signaling pathway that is involved in activation of STAT3 by CXCR4, three human breast cancer cell lines (MDA-MB-231, BT-549 and MCF-7) were analyzed for their expression of CXCR4, STAT3 and p-STAT3, using flow cytometry and western blot analysis. Flow cytometric analysis showed that CXCR4 was endogenously expressed in the cell membrane and cytoplasm of the MDA-MB-231, BT-549 and MCF-7 cells (Fig. 3A). Western blot analysis showed constitutive expression of STAT3 and p-STAT3 in all the breast cancer cell lines (Fig. 3B).
To test whether CXCR4 promotes the phosphorylation of STAT3, human breast cancer cells were treated with the CXCR4 agonist CXCL12 for 0, 2, 5, 15 and $30 \mathrm{~min}$. The expression of p-STAT3 in these cells was significantly increased at 2 to 5 min after CXCL12 treatment, and gradually declined at 15 to 30 min after CXCL12 treatment (Fig. 4A and B). Pretreatment with the CXCR4 antagonist AMD3100 inhibited the CXCL12-induced increase in the phosphorylation of STAT3 (Fig. 4C and D). These results suggest that the activation of CXCR4 promoted the expression of p-STAT3 in breast cancer cells.

STAT3 has been shown to be activated by JAK2 $(11,31)$. We then investigated whether CXCR4 mediates activation of STAT3 via JAK2. Pretreatment with the JAK2 inhibitor AG490 inhibited CXCL12-induced phosphorylation of STAT3 (Fig. 5A and B), suggesting that JAK2 mediated CXCR4induced activation of STAT3. Furthermore, we investigated whether CXCR4 directly interacted with JAK2 in MCF-7 cells, using immunoprecipitation. In the absence of CXCL12, JAK2 was immunoprecipitated with CXCR4. After CXCL12 treatment for 2 to $5 \mathrm{~min}$, the binding of JAK2 to CXCR4 was increased, accompanied by an increase in the expression of 
p-STAT3 (Fig. 5C), suggesting that CXCR4 directly activated JAK2. These results suggest that CXCR4 mediates the JAK2/STAT3 activation in breast cancer cells.

\section{Discussion}

CXCR4 and STAT3 have been known to play an important role in growth, progression, angiogenesis and metastasis of many tumor types $(3,18,32)$. In breast cancer, high CXCR4 expression has been found to be associated with poor prognosis $(2,33)$, and the CXCL12-CXCR4 signaling axis mediates breast cancer metastasis $(34,35)$. It is known that constitutively activated STAT3 is associated with breast cancer growth and metastasis $(27,28)$. In addition, it has been reported that CXCR4 contributes to murine breast cancer growth and metastasis via modulation of STAT3 (36). However, the association of CXCR4 and STAT3 has not been studied in breast cancer patients. In the present study, we investigated the expression of CXCR4, STAT3 and p-STAT3 in 208 breast cancer patients, and analyzed their correlations with clinicopathological features of these patients. We found that the expression of CXCR4, STAT3 and p-STAT3 was higher in the breast cancer tissues than that in the tumor-adjacent tissues. The combined expression levels of CXCR4/p-STAT3 were positively associated with TNM stage, tumor size, lymph node metastasis and histological grade. Furthermore, we found that CXCL12-CXCR4 induced phosphorylation of STAT3 via JAK2 in the breast cancer cell lines. Our results suggest that the CXCL12-CXCR4/JAK2/ STAT3 signaling pathway may play an important role in breast cancer malignancy and metastasis.

CXCR4 expression has been found to be upregulated in primary breast cancers, and is associated with breast cancer metastasis (37-39). Consistent with previous studies, we found that CXCR4 was highly expressed in breast cancer tissues, and the CXCR4 expression was correlated with TNM stage and lymph node metastasis. These results suggest that increased receptor numbers, at least in part, contribute to breast cancer metastasis. It has been reported that upregulation of CXCR4 receptors is induced by several oncogenic events such as hypoxia (40), HER2 overexpression (41), EGFR variant-mediated invasion (42) and TGF $\beta 1$ signaling (43), suggesting that CXCR4 mediates many oncogenic signals that lead to breast cancer metastasis. CXCL12, the CXCR4 ligand, attracts tumor cells expressing CXCR4 to organs such as the lymph node and bone marrow that produce CXCL12, thereby mediating metastasis. Our finding that CXCR4 expression is correlated with the lymph node metastasis in breast cancers highlights the important role played by CXCL12-CXCR4 signaling in breast cancer metastasis.

Several downstream signaling pathways including the JAK2/STAT3 pathway have been reported to be activated by CXCL12-CXCR4 signaling in breast cancer cells and xenograft animal models, and contribute to tumor angiogenesis, tumorigenesis and metastasis (10-12). In the present study, we found that the expression level of STAT3 was correlated with that of CXCR4, and the combined high expression of CXCR4 and STAT3, especially CXCR4 and p-STAT3, was correlated with TNM stage and lymph node metastasis of breast cancer, suggesting that the signaling pathway that involves STAT3 is critical for CXCR4-mediated breast cancer metastasis.
This finding is in accord with a previous study by Ling et al showing that STAT3 was involved in CXCR4-mediated breast cancer growth and metastasis in a xenograft animal model (36). Furthermore, we also found that the combined high expression level of CXCR4 and p-STAT3 was correlated with tumor size and histological grade. This finding is consistent with evidence that constitutively activated STAT3 participates in breast carcinogenesis in cell lines and xenograft animal models $(27,28,44)$. Our study suggests that activation of STAT3 by CXCR4 is important in human breast cancer malignancy.

In the present study, we investigated the potential signaling pathway that involves activation of STAT3 by CXCR4 in human breast cancer cell lines after treatment with CXCL12. We found that inhibition of either CXCR4 or JAK2 prevented CXCL12-induced phosphorylation of STAT3, suggesting that CXCL12-CXCR4 activated STAT3 via JAK2 in breast cancer cells. Our study agrees with previous studies that the JAK2/STAT3 pathway is activated by binding of CXCL12 to CXCR4 in human T cells (10), HEK cells expressing CXCR4 (11), and human acute lymphoblastic leukemia cells (20). However, it has been reported that CXCL12-CXCR4 cannot activate JAK2 in primary lymphocytes (21). The discrepancy may be due to different coupling mechanisms underlying activation of JAK2/STAT3 in different cells. In breast cancer cells, it is known that the JAK2/STAT3 pathway is activated by interleukin-6 $(45,46)$ and osteopontin (47). In the present study, we found that the JAK2 antagonist inhibited CXCL12-induced phosphorylation of STAT3 in three breast cancer cell lines, suggesting that the JAK2/STAT3 pathway was coupled to CXCL12-CXCR4 signaling in human breast cancer cells. Furthermore, we found that JAK2 was immunoprecipitated with CXCR4, further suggesting that CXCR4-mediated activation of JAK2 acts via direct interaction between JAK2 and CXCR4. The N-terminal part of the third intracellular loop in CXCR4, which is critical for CXCR4-mediated JAK2 activation, may be the binding site for JAK2 (11).

In summary, we found that the expression of CXCR4, STAT3 and p-STAT3 was higher in breast cancer tissues than in tumor-adjacent tissues. The expression levels of STAT3 and p-STAT3 were correlated with the expression levels of CXCR4 and clinicopathological characteristics of the breast cancer patients. The combined expression level of CXCR4 and p-STAT3 was correlated with TNM stage, tumor size, lymph node metastasis and histological grade, suggesting that activation of STAT3 by CXCR4 may play an important role in breast cancer malignancy. In addition, in breast cancer cell lines, we found that CXCL2-CXCR4 signaling activated the JAK2/STAT3 pathway. The CXCR4 or JAK2 inhibitor effectively prevented CXCL12-induced activation of STAT3. Targeting the CXCL12-CXCR4/JAK2/STAT3 signaling pathway may represent a potential therapeutic strategy for the treatment of breast cancer.

\section{Acknowledgements}

This study was supported by grants from the National Natural Science Foundation of China (no. 81173092), Liaoning S\&T Projects (no. 2011415052), and the Liaoning Province Doctor Startup Fund Program (no. 20111107). 


\section{References}

1. Lu J, Steeg PS, Price JE, et al: Breast cancer metastasis: challenges and opportunities. Cancer Res 69: 4951-4953, 2009.

2. Muller A, Homey B, Soto H, et al: Involvement of chemokine receptors in breast cancer metastasis. Nature 410: 50-56, 2001.

3. Mukherjee D and Zhao J: The role of chemokine receptor CXCR4 in breast cancer metastasis. Am J Cancer Res 3: 46-57, 2013.

4. Gil M, Seshadri M, Komorowski MP, Abrams SI and Kozbor D Targeting CXCL12/CXCR4 signaling with oncolytic virotherapy disrupts tumor vasculature and inhibits breast cancer metastases. Proc Natl Acad Sci USA 110: E1291-E1300, 2013.

5. Luker KE and Luker GD: Functions of CXCL12 and CXCR4 in breast cancer. Cancer Lett 238: 30-41, 2006.

6. Fernandis AZ, Prasad A, Band H, Klosel R and Ganju RK: Regulation of CXCR4-mediated chemotaxis and chemoinvasion of breast cancer cells. Oncogene 23: 157-167, 2004.

7. Rhodes LV, Short SP, Neel NF, et al: Cytokine receptor CXCR4 mediates estrogen-independent tumorigenesis, metastasis, and resistance to endocrine therapy in human breast cancer. Cancer Res 71: 603-613, 2011.

8. Prasad A, Fernandis AZ, Rao Y and Ganju RK: Slit proteinmediated inhibition of CXCR4-induced chemotactic and chemoinvasive signaling pathways in breast cancer cells. J Biol Chem 279: 9115-9124, 2004.

9. Liang Z, Brooks J, Willard M, et al: CXCR4/CXCL12 axis promotes VEGF-mediated tumor angiogenesis through Akt signaling pathway. Biochem Biophys Res Commun 359: 716-722, 2007.

10. Vila-Coro AJ, Rodriguez-Frade JM, Martin De Ana A, Moreno-Ortiz MC, Martinez AC and Mellado M: The chemokine SDF-1alpha triggers CXCR4 receptor dimerization and activates the JAK/STAT pathway. FASEB J 13: 1699-1710, 1999.

11. Ahr B, Denizot M, Robert-Hebmann V, Brelot A and Biard-Piechaczyk M: Identification of the cytoplasmic domains of CXCR4 involved in Jak2 and STAT3 phosphorylation. J Biol Chem 280: 6692-6700, 2005 .

12. Ling $\mathrm{X}$, Konopleva M, Zeng Z, et al: The novel triterpenoid C-28 methyl ester of 2-cyano-3, 12-dioxoolen-1, 9-dien-28-oic acid inhibits metastatic murine breast tumor growth through inactivation of STAT3 signaling. Cancer Res 67: 4210-4218, 2007.

13. Alvarez JV, Febbo PG, Ramaswamy S, Loda M, Richardson A and Frank DA: Identification of a genetic signature of activated signal transducer and activator of transcription 3 in human tumors. Cancer Res 65: 5054-5062, 2005.

14. Germain D and Frank DA: Targeting the cytoplasmic and nuclear functions of signal transducers and activators of transcription 3 for cancer therapy. Clin Cancer Res 13: 5665-5669, 2007.

15. Al Zaid Siddiquee K and Turkson J: STAT3 as a target for inducing apoptosis in solid and hematological tumors. Cell Res 18: 254-267, 2008.

16. Bromberg JF, Wrzeszczynska MH, Devgan G, et al: Stat3 as an oncogene. Cell 98: 295-303, 1999.

17. Turkson J: STAT proteins as novel targets for cancer drug discovery. Expert Opin Ther Targets 8: 409-422, 2004.

18. Wang X, Crowe PJ, Goldstein D and Yang JL: STAT3 inhibition, a novel approach to enhancing targeted therapy in human cancers (Review). Int J Oncol 14: 1181-1191, 2012.

19. Groner B, Lucks $P$ and Borghouts C: The function of Stat 3 in tumor cells and their microenvironment. Semin Cell Dev Biol 19: 341-350, 2008.

20. Soriano SF, Serrano A, Hernanz-Falcon P, et al: Chemokines integrate JAK/STAT and G-protein pathways during chemotaxis and calcium flux responses. Eur J Immunol 33: 1328-1333, 2003

21. Moriguchi M, Hissong BD, Gadina M, et al: CXCL12 signaling is independent of Jak2 and Jak3. J Biol Chem 280: 17408-17414, 2005.

22. Majka M, Drukala J, Lesko E, Wysoczynski M, Jenson AB and Ratajczak MZ: SDF-1 alone and in co-operation with HGF regulates biology of human cervical carcinoma cells. Folia Histochem Cytobiol 44: 155-164, 2006.

23. Pfeiffer M, Hartmann TN, Leick M, Catusse J, Schmitt-Graeff A and Burger M: Alternative implication of CXCR4 in JAK2/STAT3 activation in small cell lung cancer. Br J Cancer 100: 1949-1956, 2009.

24. Shen B, Zheng MQ, Lu JW, Jiang Q, Wang TH and Huang XE: CXCL12-CXCR4 promotes proliferation and invasion of pancreatic cancer cells. Asian Pac J Cancer Prev 14: 5403-5408, 2013.
25. Lee HJ, Kim SW, Kim HY, et al: Chemokine receptor CXCR4 expression, function, and clinical implications in gastric cancer. Int J Oncol 34: 473-480, 2009.

26. Diaz N, Minton S, Cox C, et al: Activation of stat 3 in primary tumors from high-risk breast cancer patients is associated with elevated levels of activated SRC and survivin expression. Clin Cancer Res 12: 20-28, 2006.

27. Ling $X$ and Arlinghaus RB: Knockdown of STAT3 expression by RNA interference inhibits the induction of breast tumors in immunocompetent mice. Cancer Res 65: 2532-2536, 2005.

28. Selander KS, Li L, Watson L, et al: Inhibition of gp130 signaling in breast cancer blocks constitutive activation of Stat 3 and inhibits in vivo malignancy. Cancer Res 64: 6924-6933, 2004.

29. Zhao L, Wang L, Jin F, et al: Silencing of estrogen receptor alpha (ERalpha) gene by promoter hypermethylation is a frequent event in Chinese women with sporadic breast cancer. Breast Cancer Res Treat 117: 253-259, 2009

30. Bai X, Jin F, Fu Y, et al: Clinicopathological significance and prognostic value of Xeroderma pigmentosum complementary group C (XPC) expression in sporadic breast cancer patients. Med Oncol 29: 1543-1553, 2012.

31. Pellegrini S and Dusanter-Fourt I: The structure, regulation and function of the Janus kinases (JAKs) and the signal transducers and activators of transcription (STATs). Eur J Biochem 248: 615-633, 1997.

32. Teicher BA and Fricker SP: CXCL12 (SDF-1)/CXCR4 pathway in cancer. Clin Cancer Res 16: 2927-2931, 2010.

33. Xu TP, Shen H, Liu LX and Shu YQ: The impact of chemokine receptor CXCR4 on breast cancer prognosis: A meta-analysis. Cancer Epidemiol 37: 725-731, 2013.

34. Jin F, Brockmeier U, Otterbach F and Metzen E: New insight into the SDF-1/CXCR4 axis in a breast carcinoma model: hypoxia-induced endothelial SDF-1 and tumor cell CXCR4 are required for tumor cell intravasation. Mol Cancer Res 10: 1021-1031, 2012.

35. Zhang M, Liu HX, Teng XD, et al: The differences in CXCR4 protein expression are significant for the five molecular subtypes of breast cancer. Ultrastruct Pathol 36: 381-386, 2012.

36. Ling X, Spaeth E, Chen Y, et al: The CXCR4 antagonist AMD3465 regulates oncogenic signaling and invasiveness in vitro and prevents breast cancer growth and metastasis in vivo. PLoS One 8: e58426, 2013.

37. Hao L, Zhang CH, Qiu YH, et al: Recombination of CXCR4, VEGF, and MMP-9 predicting lymph node metastasis in human breast cancer. Cancer Lett 253: 34-42, 2007.

38. Andre F, Xia WY, Conforti R, et al: CXCR4 expression in early breast cancer and risk of distant recurrence. Oncologist 14: 1182-1188, 2009.

39. Cabioglu N, Yazici MS, Arun BK, et al: Expression of CXCR4 predicts lymph node metastasis in early breast cancer. J Clin Oncol 22: 841s-841s, 2004

40. Schioppa T, Uranchimeg B, Saccani A, et al: Regulation of the chemokine receptor CXCR4 by hypoxia. J Exp Med 198: 1391-1402, 2003

41. Li YM, Pan Y, Wei Y, et al: Upregulation of CXCR4 is essential for HER2-mediated tumor metastasis. Cancer Cell 6: 459-469, 2004.

42. Rahimi M, George J and Tang C: EGFR variant-mediated invasion by enhanced CXCR4 expression through transcriptional and post-translational mechanisms. Int J Cancer 126: 1850-1860, 2010.

43. Zhao XP, Huang YY, Huang Y, et al: Transforming growth factor-beta1 upregulates the expression of CXC chemokine receptor 4 (CXCR4) in human breast cancer MCF-7 cells. Acta Pharmacol Sin 31: 347-354, 2010

44. Dechow TN, Pedranzini L, Leitch A, et al: Requirement of matrix metalloproteinase-9 for the transformation of human mammary epithelial cells by Stat3-C. Proc Natl Acad Sci USA 101: 10602-10607, 2004

45. Berishaj M, Gao SP, Ahmed S, et al: Stat3 is tyrosine-phosphorylated through the interleukin-6/glycoprotein 130/Janus kinase pathway in breast cancer. Breast Cancer Res 9: R32, 2007.

46. Marotta LL, Almendro V, Marusyk A, et al: The JAK2/STAT3 signaling pathway is required for growth of $\mathrm{CD} 44^{+} \mathrm{CD} 24^{-}$stem cell-like breast cancer cells in human tumors. J Clin Invest 121: 2723-2735, 2011.

47. Behera R, Kumar V, Lohite K, Karnik S and Kundu GC: Activation of JAK2/STAT3 signaling by osteopontin promotes tumor growth in human breast cancer cells. Carcinogenesis 31: 192-200, 2010. 